\title{
Characterization method of engine air behaviour from a real head-cylinder
}

\author{
Timothée ZUSSY ${ }^{1}$, Saïd ABBOUDI ${ }^{1}$, Bruno COURTALON ${ }^{2}$, Samuel GOMES $^{1}$
}

\begin{abstract}
This study is based on a head-cylinder provided by a French car manufacturer, which comes from a 4-cylinder 4-stroke spark-ignition engine. This head-cylinder adopts a Double-Over-Head-Camshaft (DOHC) with 16 valves, also two per cylinder. The purpose of this paper is to propose a characterization method of the air behaviour inside both the intake conduct of a head-cylinder and the cylinder. The cylinder stroke is known (from the cylinder capacity of the engine) as well as the valve dimensions (by measurements) but none internal dimensions of the conduct.
\end{abstract}

The proposal method follows three main steps: firstly, internal features are extracted separately by moulding with a silicon-calcium mixture, to realize the geometrical limits of the air. This step concerns only the conduct and the combustion chamber of the head-cylinder. Secondly, the two features and one of the two valves are digitized with a Faro® laser arm to compose cloud points of their shapes. Surfaces can be then reconstituted to construct a CAD model, with liftparameterized valves. Thirdly, the CAD model is exported to the CFD software Fluent ${ }^{\circledR}$ to be meshed and used to simulate air behaviour based on turbulence Reynold Stress Model (RSM) and Realizable $\kappa-\varepsilon$ models.

This last step is realized under steady-state condition, with both $2450 \mathrm{~Pa}$ and $9000 \mathrm{~Pa}$ of pressure drops between cylinder outlet and intake inlet, to obtain a view of the air motions variations in the conduct and the cylinder following the valves lift. Variations in mass flow rate results are also observed and compared with the literature for $2450 \mathrm{~Pa}$ of pressure drop, and allows to choose the best turbulence model. Those concerning $9000 \mathrm{~Pa}$ of pressure drop have been already validated with a flow bench test, in a previous study.

Keywords - Head cylinder, spark-ignition, shapes extraction, numerical, 3D, turbulence, RSM, к- $\varepsilon$.

Timothée ZUSSY, Said ABBOUDI, Samuel GOMES

ICB UMR 6303, CNRS, Département COMM, Univ. Bourgogne FrancheComté, UTBM, 90010, Belfort Cedex, France

Bruno COURTALON

R\&Dmoteurs, ZAC Technoland

25600 Brognard, France

\section{Introduction}

Numerical simulations of air behaviour in engine domain need an accurate prediction of the turbulent flow and also excellently reproducing of head cylinder's internal geometry. It has been noticed that most works of CFD in that area are based on Catia ${ }^{\circledR}$ perfect model which does not reproduce exactly the internal shapes, contrary to scanning way for instance [1]. Cases of scanning applications in automotive domain seems to be rather old in the literature.
Yau [2] described in his 1997 paper scanning process by contact measurement points to digitize complex intake port. He obtained finally an enclosed surface volume which can be transferred to commercial CAS systems through IGES translation. Chant [3] used this reverse engineering technique to determine the inlet port geometry of a diesel engine in 1998. He made a comparison between scanning external shapes obtained by silicon moulding, and direct internal scanning using machining planes off a cylinder head. He enunciated after investigations that silicon model proved to be too flexible to give completely reliable results, and digitising directly internal port proved to be more accurate, despite major drawback by lack of compatibility between analysis packages (i.e. Pro/Engineer® and scanning software here).

Concerning spatial discretization, both grid refinement and use of higher order differencing schemes improve significantly the accuracy of the predictions [4][5][6]. Polyhedral meshes constitutes an ideal compromise between structured hexahedral grids [7][8] and unstructured tetrahedral elements meshes. The first are difficult to apply without severe simplifications with complex geometries. The second require much larger number of elements and so computing time.

Due to turbulence phenomena, a structuration of the air is progressively obtain into swirl or tumble movements, according to the head conduct's geometry, the valve lift and the valve timing [9][10][11].

Escue [6] undertook a 2D numerical study of the turbulent swirling flow inside a smooth straight pipe with Fluent software, and made a comparison of $\kappa-\varepsilon$ RNG and RSM models. He also correlated his results against experimentations and concluded that RNG model was found superior for lower swirl number, and both are equivalent beyond. De Bartolo [12] presented a CFD analysis of an automobile air filter housing using RANS equations with $\kappa-\varepsilon$ Realizable turbulence model and validated her prediction results using LDA measurements. The comparison between numerical and experimental data displayed a good agreement: the mean percentage differences were lower than $1.2 \%$. This assessment permitted the characterization of the fluid dynamic behaviour of the system in those regions where the measurements were very difficult to be performed.

Perini [8] analysed the effects of different throttling strategies on intake of a single-cylinder light-duty diesel engine developed for researches, and showed good accuracy of RNG RANS model approach in capturing ensembleaveraged flow field properties. Martins [13] used equally RNG model to re-design the inlet port of a small Internal Combustion Engine in order to enhance the production of turbulence by swirl. He validated the use of this model basing on Xu's study [14], proved that the predictions displayed reasonable agreement with the experimentation in their experimental conditions. 


\section{CAD model}

\section{A. Internal shapes extraction}

As explained before, CFD simulations of air behaviour in engine domain need an excellent base of work in terms of internal shapes reproducing. It can be noticed that a headcylinder is an industrial part which include intricate orifices, internal passages with complex geometries. This can be explained the difficulty to explore them directly with a laser-arm. A method available to solve this problem is the moulding with a viscous material which keeps a certain flexibility by hardening.

The material chosen for this task was the silicon, with a low part of calcium (10\%) to give a white colour: the refraction of the scanner laser light is so optimal. The flexibility is also a significant factor, to allow to remove the moulding of the head-cylinder without damages. The mixture silicon/calcium has been placed under a vacuum to eliminate air bubbles having formed during its preparation. The obtained mouldings are so perfectly smooth. The silicone extends a little into the plans of joints, facilitating their demarcation.

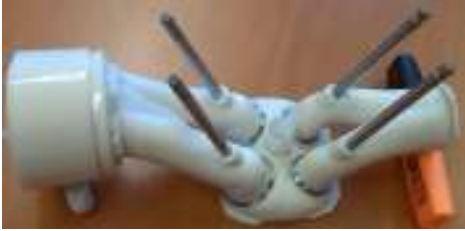

Figure 1. Silicon moulding

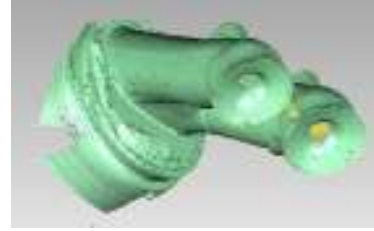

Figure 2. Cloud of points
The CAD model need three distinguished geometries: intake, exhaust and combustion chamber moulding are realized (Fig. 1). The next step is the scan of the shapes including valves, which have provided point clouds and realized by a Faro ${ }^{\circledR}$ Laser scanner arm (Fig. 2). After processing with the software Geomagic Studio ${ }^{\circledR}$, three IGES model was obtained and exported in Catia® V5R21 (Fig. 3, 4 and 5). This reverse engineering technique is faster than using CMM [15], but the IGES exit format is more difficult to treat with at Catia.

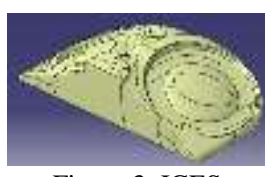

Figure 3. IGES combustion chamber

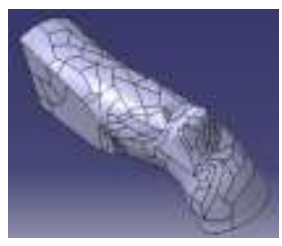

Figure 4. IGES exhaust conduct

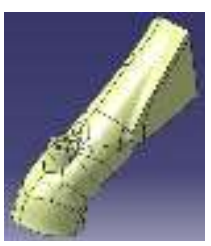

Figure 5. IGES intake conduct

\section{B. CAD reconstruction}

\section{1) Combustion chamber}

It can be observed if general shapes are correctly modelled by the laser, the edges of the valves printed on the IGES are not accurate (Fig. 3). To rebuild a complete and accurate model of the internal shapes, conducts must be placed properly in terms of the valves implant angle and centring on the combustion chamber. Valves implant angles have been found by cutting radially the combustion chamber model. Plans corresponding to angles have been added (Fig. 6 ). Concerning the centring of the valves, cylinders have been realized by approximating the borders of the printed valves on the combustion chamber (Fig. 7).

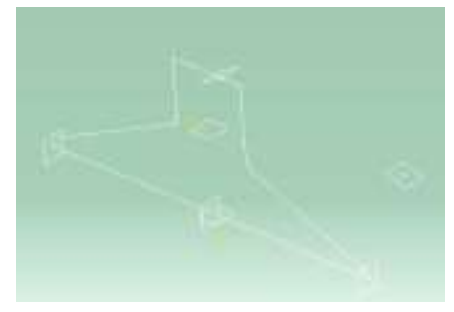

Figure 6. Radial cut of the combustion chamber

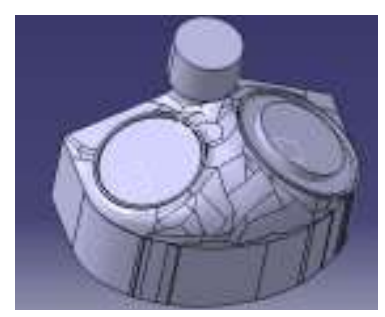

Figure 7. Combustion chamber reconstituted

\section{2) Conducts, seat valves and valves}

As before, it can be noticed that contours of the conducts at the valve seats are not accurate (Fig. 8). They were so approximated by perfect cylinders placed following the normal plane of the seat face. The centre of the conduct corresponding to that of the valve at this location were defined by this way (Fig. 9). Geometrical shapes of the valve seats were considered as a valve with no lift value. Valves (Fig. 10) were parameterized in terms of lift value, to correlate the experimental conditions of mass flow rate measurements.

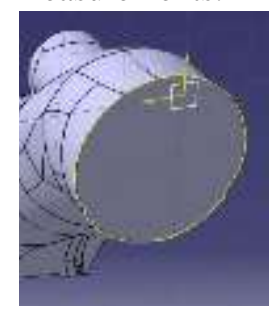

Figure 8. Valve seat contours

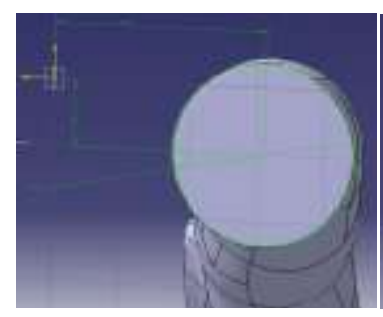

Figure 9. Centre approximation

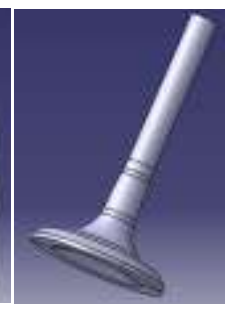

Figure 10. Valve

\section{3) Complete model of internal shapes}

Finally, the geometry consists of a combustion chamber and two main inlet and exhaust manifolds. Each one is composed by a conduct dividing itself in two parts with two valves (Fig. 11 and 12). A dummy cylinder of $0.11 \mathrm{~m}$ is placed below the cylinder head plane for the visualization of the flow field during the computations. The diameter of the cylinder added corresponds to the real engine stroke of 82 $\mathrm{mm}$.

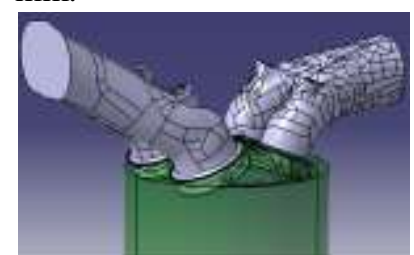

Figure 11. Complete CAD model

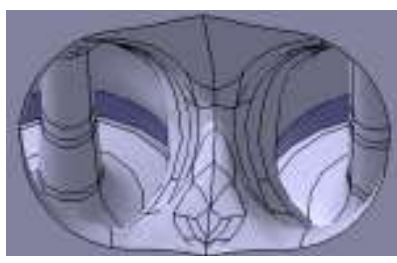

Figure 12. View from intake

\section{Computation model}

\section{A. CAD model adjustments}

A box and a specific shape were added before the real air inlet (Fig. 13). Box (arrow 1) is needed by the computation to avoid inlet dummy reversal flows and hydrodynamic effects. Indeed, the box allows to take away fluid entry. The specific shape (arrow 2) reproduces a necklace of clay put down around the intake entry during the previous experience 
in the flow bench. Part of results exploited later in this study have been indeed previously validated by experimentation. In order to extend investigations, other results based on the same geometry are compared with the literature.

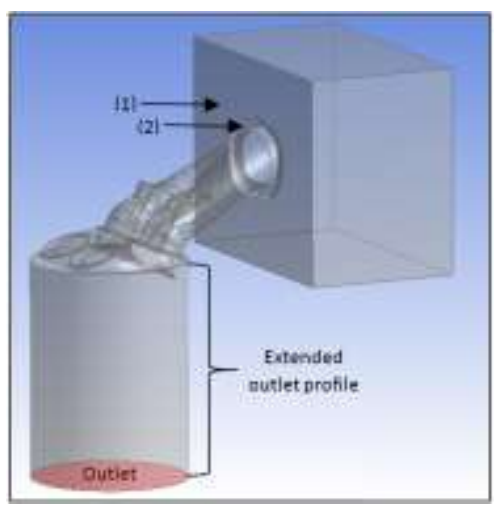

Figure 13: Complete simulation model

\section{B. Mesh}

The complexity of the computational domain requires a high density and unstructured mesh to obtain reliable computational results. The polyhedral mesh available in Fluent ${ }^{\circledR}$ results from a tetrahedral mesh transformation and allows to treat turbulence engine problems with the same accuracy than common tetra mesh while reducing computations time. From 21 million of tetra-elements, it was obtained only 4 million after transformation, including two layers of prismatic cells to take into account wall shear stresses. Binjuwair [1] carried out his study with 1.7 million of elements.

The non-equilibrium wall function available in Fluent ${ }^{\circledR}$ have been chosen, thanks to his recommendation for use in complex flows involving separation where the mean flow and turbulence are subjected to severe pressure gradients. The grid resolution is more than sufficient for the use of the non-equilibrium wall function $(30<y+<300)$ and do not need the use of a second-order discretization scheme.

\section{Turbulence model choice}

The calculations were realized by solving compressible Navier-Stokes equations for mass, momentum, energy and turbulence equations for $\kappa-\varepsilon$ and RSM models.

A previous study was carried out under a pressure drop of $9000 \mathrm{~Pa}$ with the head cylinder considered. The results of mass flow rate were validated by correlation with experimental data, furnished by a flow bench.

A second study was performed this time without experimental validation, to test the model by considering the literature. That is why the same boundary conditions than those whom Binjuwair used were applied. There are shown in Table I. However, geometrical shapes are not the same: the cylinder dimensions and the valve diameter used in his work are higher than those utilized in the present study. To maintain such consistency in case of comparison, mass flow rate values obtained by Binjuwair in equal pressure drop and lift value must be higher too.

It can be seen in Table II a problem considering the $\kappa-\varepsilon$ model values: the results are not coherent. The mass flow rate values obtained here for $8 \mathrm{~mm}$ and $9.45 \mathrm{~mm}$ valve lift exceed Binjuwair's values (respectively $+9 \%$ and $+2 \%$ ) while by definition, the section of passage of air of the conduct he applied, is higher than the one that was carried out in this study. On the other hand, for $6 \mathrm{~mm}$ lift value, mass flow rate value respects this condition. But there is an excess splits of mass flow rate regard to what is expected.

RSM model produces the best results, with a progressive increase of the gap relative to the lift value. However, the gap seems to be relatively constant without anomaly of coherence such as the $к-\varepsilon$.

TABLE I. ENGINE PARAMETERS

\begin{tabular}{|c|c|c|c|}
\cline { 2 - 4 } \multicolumn{1}{c|}{} & Binjuwair & This study & Gap \\
\hline $\begin{array}{c}\text { Cylinder } \\
\text { capacity }\left(\mathrm{cm}^{3}\right)\end{array}$ & 500 & 397 & $+21 \%$ \\
\hline $\begin{array}{c}\text { Valve } \\
\text { diameter }(\mathrm{mm})\end{array}$ & 35.5 & 31.3 & $+12 \%$ \\
\hline
\end{tabular}

TABLE II: COMPARISON OF MASS FLOW RATE $\boldsymbol{m}$ VALUES

\begin{tabular}{|c|c|c|c|c|c|}
\hline & RSM & & $\kappa-\varepsilon$ & \multirow{2}{*}{$\begin{array}{c}\text { Gap } m \\
\text { RSM } \\
\text { Binjuwair/ } \\
\text { RSM }\end{array}$} & \multirow{2}{*}{$\begin{array}{c}\text { Gap } m \\
\text { RSM } \\
\text { Binjuwair/ } \\
\kappa-\varepsilon\end{array}$} \\
\hline Lifts & $m$ Binjuwair & \multicolumn{2}{|c|}{$\dot{m}$ of this study } & & \\
\hline $6 \mathrm{~mm}$ & 0.062 & 0.047 & 0.035 & $-24 \%$ & $-43 \%$ \\
\hline $8 \mathrm{~mm}$ & 0.077 & 0.053 & 0.085 & $-31 \%$ & $+9 \%$ \\
\hline $9.5 \mathrm{~mm}$ & 0.134 & 0.100 & 0.136 & $-25 \%$ & $+2 \%$ \\
\hline
\end{tabular}

\section{Iv. Numerical flow structures}

An analysis of flow structures obtained by several computations is presented in this section. Tumble and swirl motions are obtained respectively by vertical and horizontal planes.

As valve lift $\mathrm{L}_{\mathrm{v}}$ increases, three separates stages to the flow area development are distinguished [16]: the head of valve influences less and less the passage area and so the behaviour of the fluid in the cylinder. The valve gap area were thus established for intake case: the first stage corresponding to low valve lift is composed of each lift values $0 \mathrm{~mm}<\mathrm{L}_{\mathrm{v}} \leq 4.25 \mathrm{~mm}$, the second stage is constituted of these following lift values: $4.25 \mathrm{~mm}<\mathrm{L}_{\mathrm{v}} \leq 8.5 \mathrm{~mm}$. Finally, the third case which is the high-opened stage concerns all lift values of $\mathrm{L}_{\mathrm{v}}>8.5 \mathrm{~mm}$ (Fig. 15).

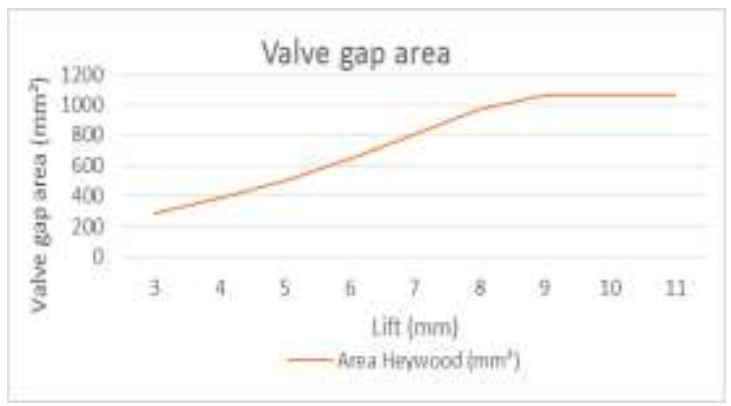

Figure 14: Valve gap area

Two cases of lift from the second and one from the third stage are treated in this study: both $6 \mathrm{~mm}$ and $8 \mathrm{~mm}$ included in the second stage and $9.45 \mathrm{~mm}$ corresponding to the maximum lift of the engine, included in the third stage. The first stage is not considered here because the results are not sufficiently reliable qualitatively. Following studies can be validate thanks to the literature through a paper made by Binjuwair in 2013. 


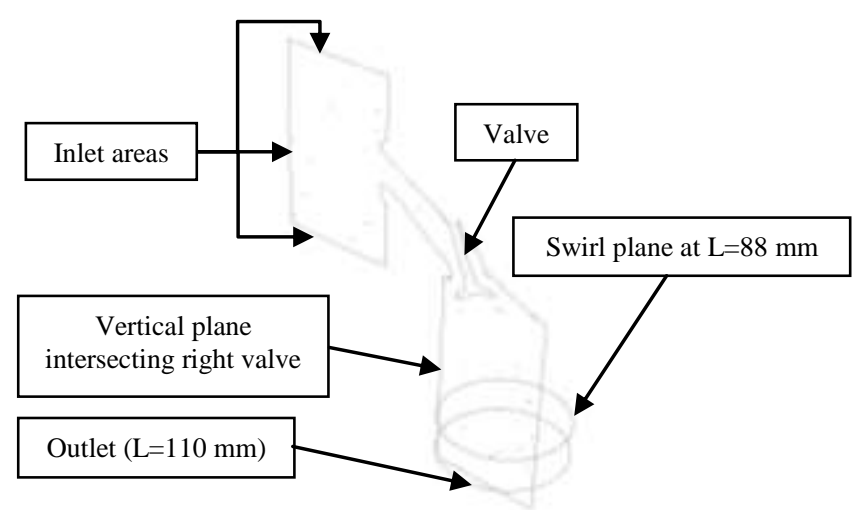

Figure 15: Location of characteristic planes studied

\section{A. Tumble motions}

To handle this subject, a vertical plane is created in the in-cylinder mesh (Fig. 15). It is located in the cylinder, intersected the right valve to show air behaviour around and below the valve, according to a radial plane.

\section{1) Effect of valve lift increasing}

For the second stage, which is an intermediate lift level according to [1], $6 \mathrm{~mm}$ and $8 \mathrm{~mm}$ were explored.

At this stage and under $2450 \mathrm{~Pa}$, Fig. 16 and Fig. 17 show that the valve is sufficiently opened allowing the air flow to begin to come off the cylinder side: centre of the vortex moves away from the cylinder wall, towards the vertical axis of the cylinder. Speed also becomes more important adjacent to the cylinder wall, but its magnitude stayed roughly the same.

Tumble is created partly thanks to the head of the valve. There are more frictions but the speed increases inside the cylinder following the lift value, due to the widest area of air across the valve and the turbulent energy inside the conduct. Thus really expands its diameter and the filling is improved.

For both cases, the vortex presents rather a round shape, and the counter-tumble is not really developed. The area of the cylinder below the valve is partially fill and the air speed located here is more important than that of the tumble itself. This phenomena may be caused by the effect of reverse flow after hitting the wall.

\section{2) Effect of pressure drop increasing}

The intermediary lift value of $8 \mathrm{~mm}$ is considered here (Fig. 17 and Fig. 18). By increasing the pressure drop across the cylinder head from 2450 to $9000 \mathrm{~Pa}$ in our case, variations in tumble flow structures can be observed following the model used.

Computations were realized with the same boundary conditions between the two models. It can be noticed that the velocity increases evidently and as a consequence the vortex grows and moves far away from the wall, toward the cylinder axis. The filling of the cylinder is almost perfect because the vortex is now located under the cylinder and its dimensions changes: it strongly grew up.

To make a comparison with the three stages previously determined, $8 \mathrm{~mm}$ is very close to the third stage where the valve head does not slow down any more the passage of the fluid. However, it involves in the growth of the tumble and the counter-tumble. The behaviour of the vortex is similar here to an increase of the valve lift, as explained before.
For the third stage, Fig. 19 shows the behaviour of the fluid when the valve is $9.45 \mathrm{~mm}$. At this stage, intake valve head influences no more the entry of the fluid to the combustion chamber quantitatively. Only the tail of the valve can disturb the filling, but it is small-dimension compared to the size of the conduct. Qualitatively, it can be observed that the flow does not need to hit the wall to create a tumble, and this is the head of the valve which trains its construction. Velocity value is high around the tumble and contributes to maintain it round shape inside the cylinder. High velocity region forever exists close to the cylinder wall, it becomes more important as the valve opens, but it concerns now all the cylinder. This is due to the countertumble which is full-developed and contributes to improve still the filling under the valve.

\section{B. Swirl motions}

To handle this subject, two horizontal planes are created in the in-cylinder mesh (Fig. 15). They are located far the head-cylinder gasket plane, to show air behaviour inside the cylinder according to an axial plane.

\section{1) Effect of valve lift increasing}

Figures 20 and 21 show the variations of the behaviour of the fluid following two lift values: $6 \mathrm{~mm}$ and $8 \mathrm{~mm}$ at $\mathrm{L}=88$ $\mathrm{mm}$. Valves are located in the lower zone of the considered area. It can be seen an increase of the general velocity magnitude through the cylinder area when the lift is increased.

Moreover, the two vortex does not have a symmetrical repartition on the first case, and swirl are concentrated on the upper extremity of the cylinder, away from the valves.

As the lift increases, swirl vortex grows up and their centres move away to stabilize them along a line crossing horizontally the cylinder in its middle. Furthermore, for 8 $\mathrm{mm}$ lift value, the cylinder is divided in two separate areas by a high velocity zone. This phenomenon can be due to the encounter of the two jets appearing from valves located from part and others of this zone. It can be noticed an additional relative high velocity everywhere in the cylinder area except in the centre of the vortex.

\section{2) Effect of pressure drop increasing}

Fig. 22 and Fig. 23 show the variations of the behaviour of the fluid following a strong increase of pressure drop from $2450 \mathrm{~Pa}$ to $9000 \mathrm{~Pa}$. The distance between the head cylinder plane is chosen at $110 \mathrm{~mm}$ to respect the same condition as Binjuwair. $110 \mathrm{~mm}$ length is equally chosen because the performance of the geometrical shapes of the conduct and the valve can also be tested by this way: this observation shows the capacity of maintaining homogenization of the mixture fuel/air inside the cylinder trough swirl movements.

If we consider that the real stroke of this engine is 82 $\mathrm{mm}$, it can be observed that swirls exist all the time of the stroke. The pressure drop seems to have no influence concerning the vortex formation, even if the velocity increases strongly. It can be observed an expansion of the high velocity area in the centre of the cylinder and a little displacement of the centre of the vortex toward the horizontal line mentioned in the previous paragraph. 
Proc. of the Seventh International Conference On Advances in Mechanical, Aeronautical and Production Techniques - MAPT 2017 Copyright (c) Institute of Research Engineers and Doctors. All rights reserved.

ISBN: 978-1-63248-129-0 doi: 10.15224/ 978-1-63248-129-0-45

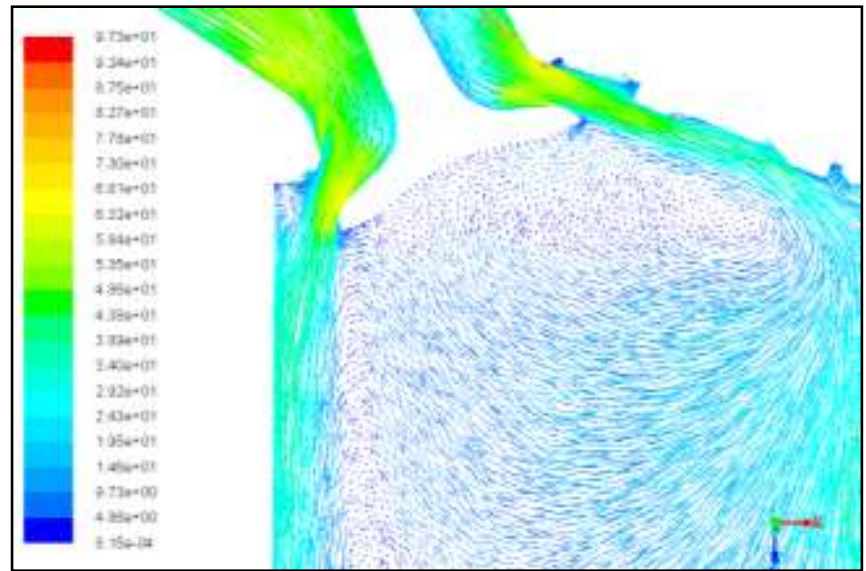

Figure 16. Tumble with $\mathrm{Lv}=6 \mathrm{~mm}, 2450 \mathrm{~Pa}$

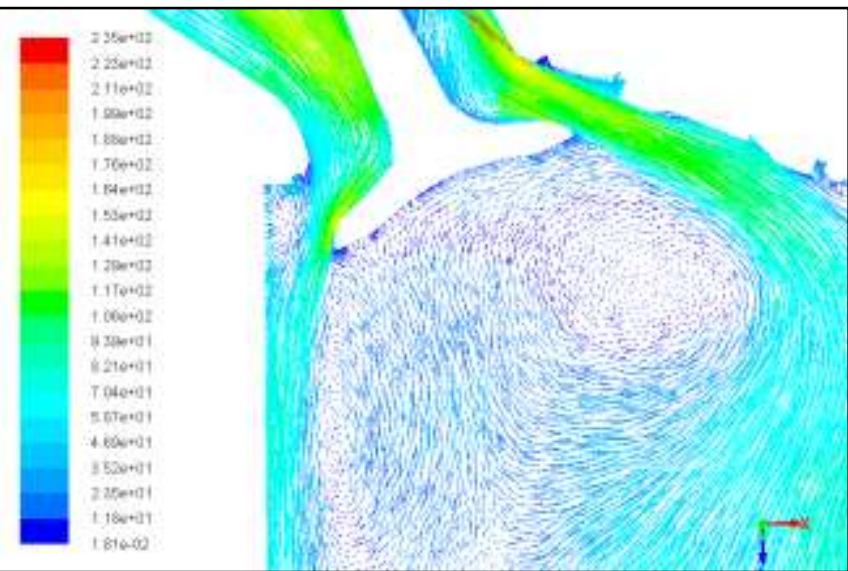

Figure 18. Tumble with Lv $=8 \mathrm{~mm}, 9000 \mathrm{~Pa}$
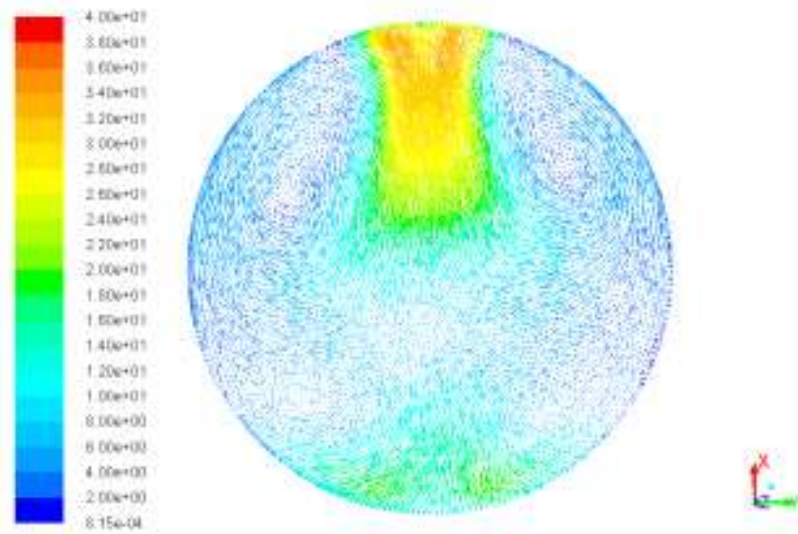

Figure 20. Swirl at $\mathrm{L}=88 \mathrm{~mm}, \mathrm{Lv}=6 \mathrm{~mm}, 2450 \mathrm{~Pa}$
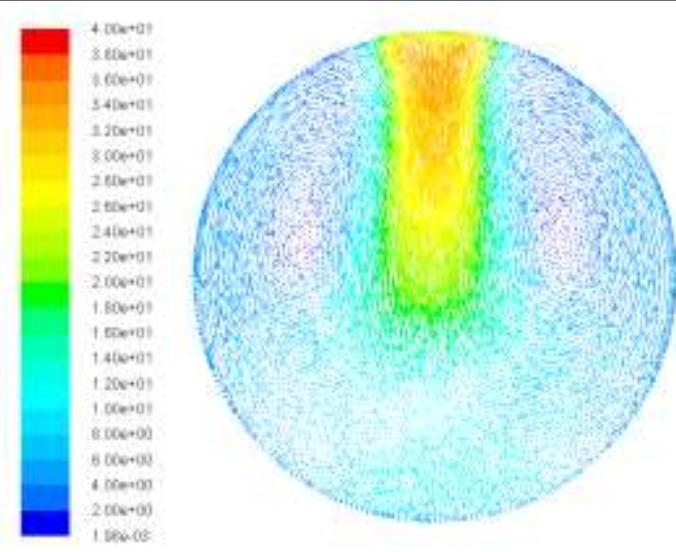

$t^{x}-2$

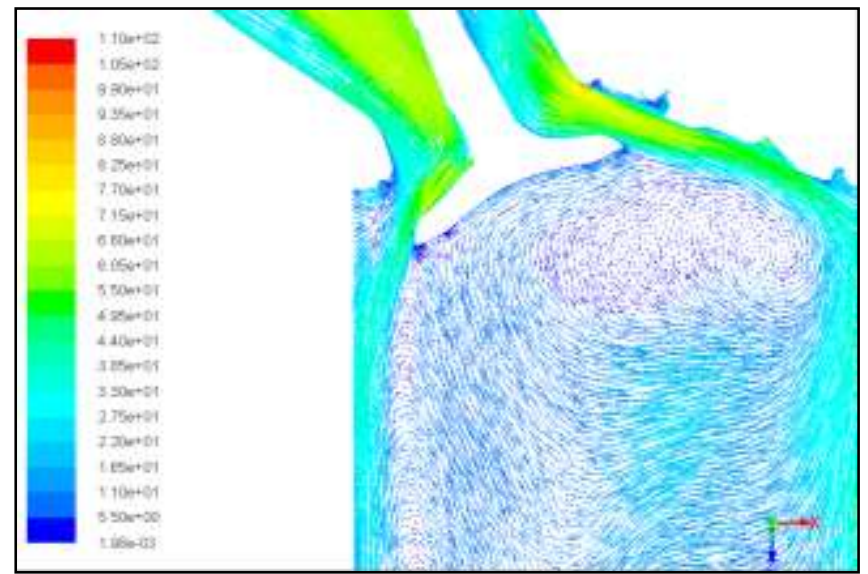

Figure 17. Tumble with Lv $=8 \mathrm{~mm}, 2450 \mathrm{~Pa}$

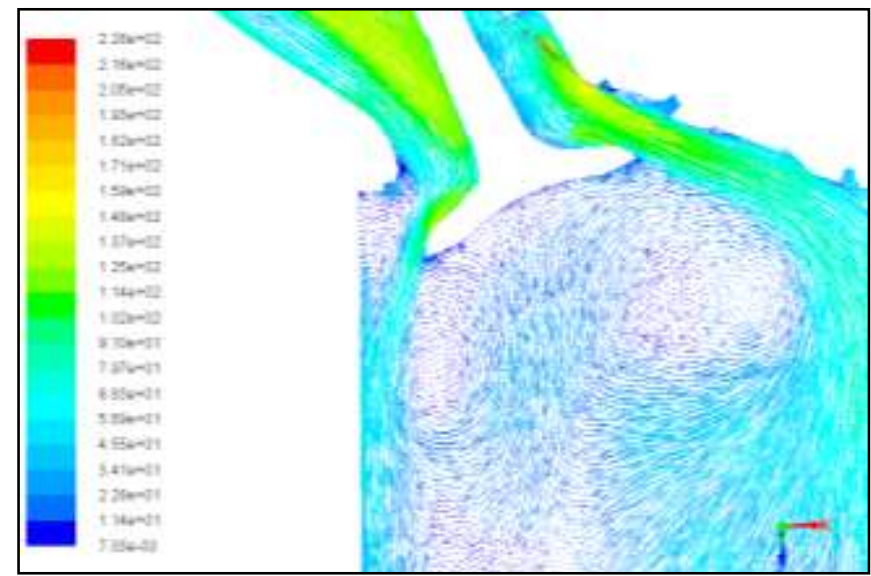

Figure 19. Tumble with $\mathrm{Lv}=9.45 \mathrm{~mm}, 9000 \mathrm{~Pa}$

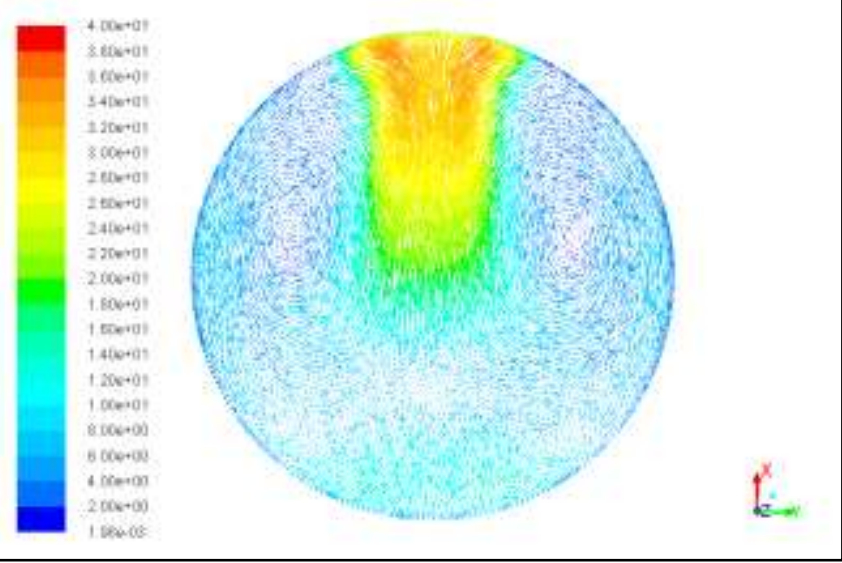

Figure 21. Swirl at $\mathrm{L}=88 \mathrm{~mm}, \mathrm{Lv}=8 \mathrm{~mm}, 2450 \mathrm{~Pa}$

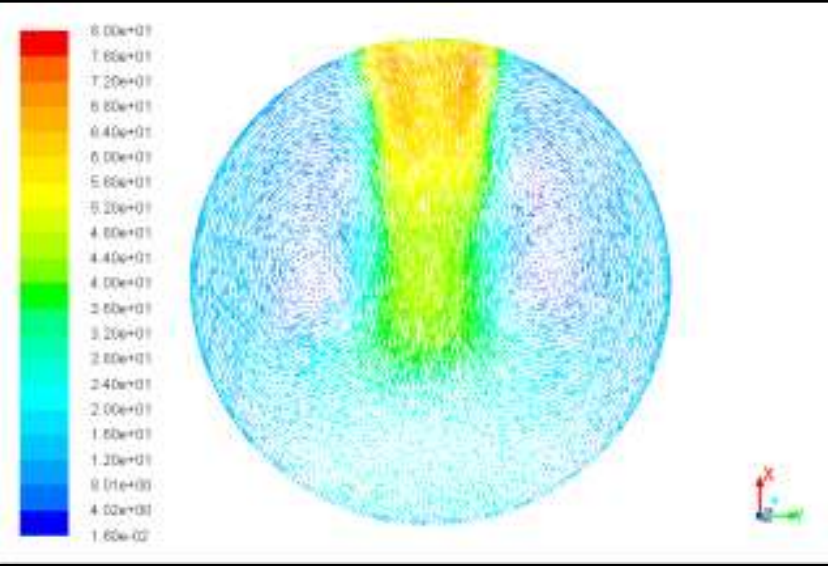

Figure 23: Swirl at $\mathrm{L}=110 \mathrm{~mm}, \mathrm{Lv}=8 \mathrm{~mm}, 9000 \mathrm{~Pa}$ 


\section{v. Conclusion}

Investigations of this previous study were related to obtaining mass flow rate results and phenomena views inside the conduct and the cylinder of a spark-ignition engine. Only the head-cylinder from the engine was available as a working basis.

Internal shapes were extracted by moulding and were reconstructed as a CAD model. This reconstruction was based on a cloud of points obtained thanks to a laser arm.

This model was then exported in the software Fluent ${ }^{\circledR}$ to realize its mesh and turbulence computations. The mesh considered was a polyhedral type.

The numerical analysis was employed firstly to choose a turbulence model between the RSM and the Realizable $\kappa-\varepsilon$. A quick comparison of the mass flow rate results was realized with a similar case of the literature by using the same boundary conditions. It appears that the RSM model is the best to apply with this engine application.

Other correlations were made with the literature to control qualitatively both swirl and tumble motions formed within the cylinder. Three planes were created inside the mesh: a vertical plane which cut the cylinder into a radial plane, across the right valve to visualize tumbles, and two others horizontal in $\mathrm{L}=88 \mathrm{~mm}$ and $110 \mathrm{~mm}$ from the head cylinder plane, to visualize swirls. In case of increasing the valve lift, the flow structures remain similar as in Binjuwair's [1] study, but it can be added that the main tumble is created partly thanks to the head of the valve, and it grows up following the increase of the lift value and so the velocity. It moves far away from the cylinder wall, towards the cylinder axis and under the valve. The filling is so improve as soon as lift grows. The counter-tumble develops itself equally. In case of increasing pressure drop, it was found that the same phenomena happens: vortex grows up strongly and moves to the cylinder axis.

The general pattern of the scenes remain similar in terms of phenomena being able to be seen in Binjuwair's study. It can be noticed that he obtained a best filling of the cylinder for $6 \mathrm{~mm}$ lift value, mainly thanks to a wider passage of air related to both higher dimensions of valve and cylinder. Its velocity is substantially greater for the same reasons.

\section{Acknowledgment}

Timothee ZUSSY thanks the organism "Pays de Montbéliard Agglomérations" which funded these researches and the company $\mathrm{R} \&$ Dmoteurs which provided experimental results.

\section{References}

S. Binjuwair, S. Ibrahim, G. Wigley, and G. Pitcher, "Characterisation of flow structures inside an engine cylinder under steady state condition," World J. Eng., vol. 10, no. 3, 2013.
H.-T. Yau, "Reverse engineering of engine intake ports by digitization and surface approximation," Int. J. Mach. Tools
Manuf., vol. 37, no. 6, pp. 855-871, Jun. 1997.

A. Chant, D. Wilcock, and D. Costello, "The determination of IC engine inlet port geometries by reverse engineering," Int. J. Adv. Manuf. Technol., vol. 14, no. 1, pp. 65-69, Jan. 1998.

[4] C. Rumsey and T. Beutner, Introduction: Computational Fluid Dynamics Validation for Synthetic Jets, vol. 44, no. 2. 2006.

[5] H. Mohamed Niyaz and A. S. Dhekane, "Twin Helical Intake Port Design Optimization And Validation By Using CFD Analysis," Int. J. Emerg. Technol. Adv. Eng., vol. 4, no. 4, pp. 454-462, 2014.

[6] A. Escue and J. Cui, "Comparison of turbulence models in simulating swirling pipe flows," Appl. Math. Model., vol. 34, no. 10, pp. 2840-2849, Oct. 2010.

[7] B. Semlitsch, Y. Wang, and M. Mihăescu, "Flow effects due to valve and piston motion in an internal combustion engine exhaust port," Energy Convers. Manag., vol. 96, pp. 18-30, May 2015

[8] F. Perini, P. C. Miles, and R. D. Reitz, "A comprehensive modeling study of in-cylinder fluid flows in a high-swirl, lightduty optical diesel engine," Comput. Fluids, vol. 105, pp. 113124, Dec. 2014.

[9] A. M. Bharadwaj, K. Madhu, J. Seemanthini, K. G. Vismay, S. M. Anand, and S. Arvind, "Study of Swirl and Tumble Motion using CFD," IJTARME, vol. 2, no. 1, pp. 36-39, 2013.

[10] W. Zhijun and H. Zhen, "In-cylinder swirl formation process in a four-valve diesel engine," Exp. Fluids, vol. 31, no. 5, pp. 467473, Nov. 2001

[11] V. Iorga-Siman, "Etude par simulation numerique des ecoulements dans le conduit d'admission d'un moteur a levee de soupape d'admission variable," Conservatoire National des Arts et Métiers, 2012.

[12] C. De Bartolo, A. Algieri, and S. Bova, "Simulation and experimental validation of the flow field at the entrance and within the filter housing of a production spark-ignition engine," Simul. Model. Pract. Theory, vol. 41, no. 41, pp. 73-86, Feb. 2014.

[13] J. Martins, S. F. C. F. Teixeira, and S. Coene, "Design of an inlet track of a small I.C. engine for swirl enhancement." Associação Brasileira de Engenharia e Ciências Mecânicas (ABCM), 01Nov-2009.

[14] J. Xu, Q. Yao, X. Cao, and K. Cen, “Algebraic stress model with RNG $\varepsilon$-equation for simulating confined strongly swirling turbulent flows," J. Therm. Sci., vol. 10, no. 1, pp. 14-19, Mar. 2001.

[15] M. Dúbravčík and Š. Kender, "Application of Reverse Engineering Techniques in Mechanics System Services," Procedia Eng., vol. 48, pp. 96-104, 2012.

[16] J. B. Heywood, Internal Combustion Engine Fundamentals, McGraw-Hil. 1989.

About Author (s)

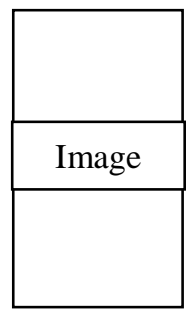

[Type a brief summary of author details.] 